\title{
Free vibration analysis of cracked plates using peridynamics
}

\author{
Jeeyeon $\mathrm{Heo}^{\mathrm{a}}$, Zhenghao Yang ${ }^{\mathrm{a}}$, Wenxuan Xia ${ }^{\mathrm{a}}$, Selda Oterkus ${ }^{\mathrm{a}}$ and Erkan \\ Oterkus $^{\mathrm{a}^{*}}$
}

${ }^{a}$ PeriDynamics Research Centre, University of Strathclyde, 100 Montrose Street Glasgow G4 0LZ, United Kingdom

\begin{abstract}
In this study, free vibration analysis of cracked plates was performed by using peridynamics. Peridynamics is a new continuum mechanics formulation which is especially suitable for problems including discontinuities such as cracks. A peridynamic Mindlin plate formulation was used and the numerical implementation was done using commercial finite element software, ANSYS. First, the formulation was verified by considering intact and cracked plates and peridynamic solutions were compared against numerical, theoretical and experimental results available in the literature. Once the formulation was verified, the effect of plate thickness, crack size and crack orientation on the natural frequencies were investigated for a centrally cracked plate. It was found that natural frequency values increase as plate thickness increases. On the other hand, increase in crack length decreases the natural frequency values. Moreover, crack orientation also increases natural frequencies for larger cracks. Finally, linear variation of thickness inside the plate causes a tilt of the mode shapes towards the thin side of the plate.
\end{abstract}

Keywords: Free vibration; crack; plate; peridynamics

\section{Introduction}

Ships and offshore structures are subjected to harsh marine environment which can cause several damage scenarios including fracture and corrosion. Ultrasonic damage detection techniques are effective and increasingly popular techniques for damage detection. Cracked structures can demonstrate different free vibration behaviour in terms of natural frequencies and mode shapes with respect to their intact form. Therefore, investigation of free vibration behaviour of cracked structures can provide invaluable information for damage detection techniques.

There are numerous studies in the literature focusing on free vibration behaviour of structures, especially plate type structures. Amongst these Stahl and Keer (1972) studied eigenvalue problem of cracked rectangular plates to determine their natural frequencies and mode shapes. They formulated the problem as dual series equations and reduced to homogeneous Fredholm integral equations of the second kind. Krawczuk (1993) developed a finite element model of a rectangular plate with a through crack to investigate the effect of crack location and its length on the natural frequency of simply supported and cantilever plates. Qian et al. (1990) also used finite element method to determine natural frequencies for a simply-supported square plate and a cantilever plate with a thorough crack. They also performed an experimental study to verify their numerical results. Zeng et al. (2016) performed the vibration analysis of a rectangular plate with a side crack based on classical thin plate theory using the Ritz method with admissible functions that were constructed by the moving least square method with enriched basis functions. They investigated the effect of location, length and orientation of side cracks on vibration frequencies and mode shapes of cracked plates. Doan et al. (2019) recently studied free vibration analysis of cracked Mindlin plates by using phase-field method. Soni et. al. (2018) developed an analytical model for vibration analysis of partially cracked plates coupled with fluid medium. Huang et. al. (2011) utilized

\footnotetext{
* Corresponding author. Tel.: +44-141-548-3876.

E-mail address:erkan.oterkus@strath.ac.uk
} 
Ritz method to accurately determine the frequencies of thick and crack plates based on Mindlin plate theory. Nguyen-Thoi et. al. (2014) used cell-based smoothed discrete shear gap method for free vibration analysis of cracked Mindlin plates by utilizing the crack-tip singular enriched functions of the extended finite element method. In another study, Yang et. al. (2017) proposed an extended edge-based smoothed discrete shear gap method for free vibration analysis of cracked Reissner-Mindlin plate by implementing the edge based strain smoothing operation into the discrete shear gap-based extended finite element method. Joshi et. al. (2017) developed a non-classical analytical model for free vibration of cracked rectangular isotropic and functionally graded micro-plates in the presence of thermal environment by combining classical plate theory and modified couple stress theory. Hosseini-Hashemi et. al. (2010) analyzed free vibration of moderately thick rectangular plates with an arbitrary number of all-over partthrough cracks by using a set of exact closed-form characteristic equations incorporating shear deformation and rotary inertia. Joshi et. al. (2015) developed an analytical model for free vibration analysis of a thin rectangular isotropic plate containing an internal crack located at the center of the plate using classical plate theory. Xu and Chen (2017) proposed a universal method combining differential quadrature finite element method and virtual spring technique to analyze free vibration of thin plates with irregular cracks. Shahverdi and Navardi (2017) presented an elemental approach based on differential quadrature method for free vibration analysis of cracked thin plate structures. Gupta et. al. (2018) performed free vibration analysis of square center-cracked plate at various orientations using finite element method. Azam et. al. (2017) investigated the free vibration analysis of rhombic plates with a preexisting central crack using finite element method and Mindlin plate theory. Nguyen-Thanh et. al. (2018) developed an approach which couples the meshfree method and isogeometric analysis for free-vibration analysis of cracks in thin-shell structures. Zhang (2017) adopted discrete singular convolution method for free vibration analysis of cracked rectangular plates. Xue and Wang (2019) studied the free vibration of a flat plate with a side crack of Mode I fracture reinforced by one stiffener parallel to the edges of the plate. Yu et. al. (2019) introduced a new approach using extended finite element method to significantly improve the accuracy of natural frequency in free vibration analysis of cracked Reissner-Mindlin plates.

In this study, an alternative computational method, peridynamics, was utilised to perform free vibration analysis of cracked plates. Peridynamics (PD) was introduced by Silling (2000) to overcome the limitations of Classical Continuum Mechanics (CCM). Equations of motion of PD theory are in integro-differential equation form and they do not contain spatial derivatives which allow analysing discontinuities such as cracks without applying any special treatment to obtain the solution. There has been an increasing number of studies on peridynamics, especially during last few years. Amongst these Imachi et. al. (2019) introduced a new transition bond method within ordinary-state based peridynamics framework for dynamic fracture analysis. Javili et. al. (2019) developed a novel continuum-kinematics inspired approach for peridynamics. Nguyen and Oterkus (2019) developed a novel bond based peridynamic model for three-dimensional complex beam structures with six degrees of freedom based on Timoshenko beam theory. Wang et. al. (2019) proposed a coupled peridynamic-finite element method for modelling mechanical behavior and damage growth in materials. Ni et. al. (2019) introduced two new implicit static solution procedures to study crack propagation problems by adopting peridynamics-based numerical tool. Ozdemir et. al. (2020) utilized peridynamics to simulate dynamic wave propagation and crack propagation in functionally graded materials. Li et. al. (2020) developed a peridynamic model to investigate thermally-induced fracture for cubic polycrystals and ceramic made of different materials. Imachi et. al. (2020) performed dynamic fracture analysis for the crack arrest phenomenon by using ordinary-state based peridynamics and transition bond concept. Guski et. al. (2020) investigated the 
damage behavior of a 2D microstructure taken from a plasma sprayed ceramic coating used in solid oxide fuel cell sealing systems by using peridynamics. Ye et. al. (2019) performed peridynamic simulations of submarine surfacing through ice. Vazic et. al. (2020) investigated in-plane and out-of-plane failure of an ice sheet using peridynamics. Wang et. al. (2020) determined the horizon size for state-based peridynamics. An extensive review of peridynamics research can be found in Javili et. al. (2019).

There are fundamental differences between peridynamics and some other numerical techniques used for fracture predictions including smoothed particle hydrodynamics (SPH) and extended finite element method (XFEM). Although SPH uses governing equations similar to peridynamics as well as an influence region similar to the horizon concept in peridynamics, foundations of SPH are different than peridynamics. SPH concept is based on the definition of the delta functional widely used in mathematics. SPH introduces a smoothing concept for the delta function so that the value of a function at a particular point can be obtained by partial contributions of the values of the function at other points inside an influence region. In other words, the value of a function at a point can be obtained by performing interpolation which makes it a purely numerical approach. On the other hand, as explained in the next section, peridynamics is a non-local continuum mechanics formulation, rather than a purely numerical approach as in SPH, which takes into account non-local interactions between material points inside horizon (influence region) and governing equations can be obtained from Euler-Lagrange equations by calculating the total kinetic and potential energies of the system. XFEM was introduced to overcome the mesh dependency problem of cohesive zone models by allowing splitting of regular finite elements. Although this is a promising idea, it is not very suitable to deal with large number of cracks interacting with each other. Moreover, detection of the crack surface in 3-Dimensional analysis is a major challenge for XFEM. On the other hand, cracks are not treated as special objects in peridynamics and they are part of the formulation. Therefore, any number of cracks can occur and interact with each other in peridynamics. There is no need to define crack growth laws in peridynamics for the crack evolution about when it will start to grow, in which direction it will grow, whether it should branch or not. There is also no need to detect the crack surfaces. Hence, 3-Dimensonal models do not introduce any additional challenge to the computations.

In this study, Mindlin plate peridynamic formulation developed by Diyaroglu et al. (2015) was used to formulate the problem. Numerical implementation was done using commercial finite element software, ANSYS, by following the approach described in Yang et. al. (2018). Such an approach provides a straightforward way to determine the natural frequencies and mode shapes by using the modal analysis algorithms already implemented in ANSYS software.

\section{Peridynamic theory}

\subsection{Basic Theory}




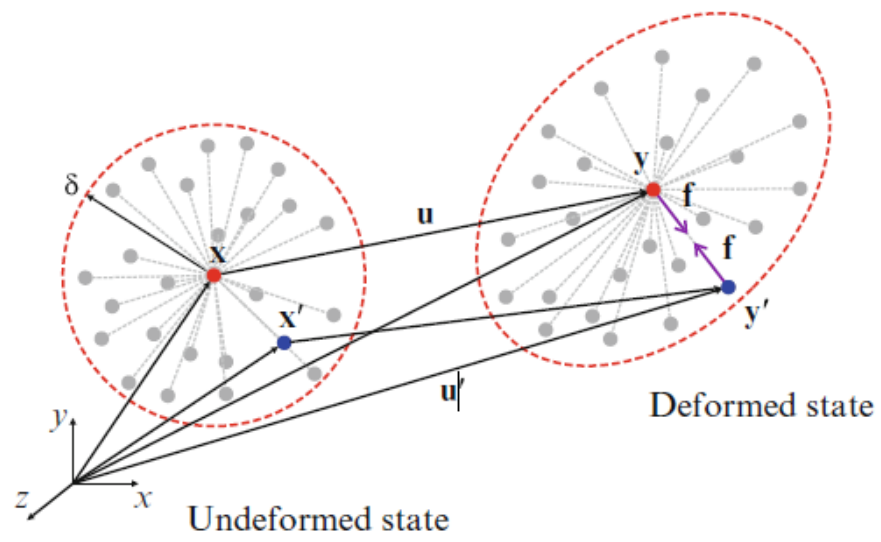

Fig. 1. Undeformed and deformed state of peridynamic material points (Madenci and Oterkus, 2014).

Peridynamic theory is a new continuum mechanics formulation introduced by Silling [1] to overcome the limitations of classical continuum mechanics (CCM). The most prominent feature of the PD theory is that it replaces the spatial differential term of the equation of motion with an integral term. PD theory is based on the traditional continuum assumption where the structure consists of infinitely small volumes called material points. PD theory is within the class of non-local continuum mechanics in which material points can interact with each other in a non-local manner as long as they are located within their domain of influence named as horizon, $\delta$. The material points that belong to the horizon of a particular material point are called family members As shown in Fig. 1, in the original bond-based PD formulation that Silling (2000) proposed, it is assumed that the interaction force between the two material points is equal and opposite.

\subsection{Equation of Motion}

The peridynamic equation of motion can be derived from Lagrange's equation. Therefore, the peridynamic equation of motion for the material point $k$ can be written as

$$
\frac{d}{d t}\left(\frac{\partial L}{\partial \mathbf{q}_{(k)}}\right)-\frac{\partial L}{\partial \mathbf{q}_{(k)}}=0
$$

where Lagrangian $(L)$ is defined as the difference between the total kinetic energy of the system, $T$, and the total potential energy of the system, $U$. The total kinetic energy and total potential energy of the system can be calculated by integrating the kinetic energy and potential energy of all material points. Substituting Lagrangian, $L$, in Eq. (1) and using the assumption for bond-based peridynamics mentioned earlier, the peridynamic equation of motion can be obtained as

$$
\rho \mathbf{u}(\mathbf{x}, t)=\int_{H_{\mathbf{x}}} \mathbf{f}\left(\mathbf{u}\left(\mathbf{x}^{\prime}, t\right)-\mathbf{u}(\mathbf{x}, t), \mathbf{x}^{\prime}-\mathbf{x}\right) d V^{\prime}+\mathbf{b}(\mathbf{x}, t)
$$

in which $\rho$ is the mass density, $H_{\mathbf{x}}$ represents the neighbourhood region within the horizon of the material point $\mathbf{x}, \mathbf{u}$ and $\mathbf{x}$ are displacement and position vectors, respectively, and $\mathbf{b}$ represents the body force density exerting on the material point $\mathbf{x}$. Moreover, $\mathbf{f}$ is the PD force density vector of the pairwise interaction between material points which can be expressed as 


$$
\mathbf{f}\left(\mathbf{u}^{\prime}-\mathbf{u}, \mathbf{x}^{\prime}-\mathbf{x}\right)=c s \frac{\mathbf{y}^{\prime}-\mathbf{y}}{\left|\mathbf{y}^{\prime}-\mathbf{y}\right|}
$$

with

$$
s=\frac{\left|\mathbf{y}^{\prime}-\mathbf{y}\right|-\left|\mathbf{x}^{\prime}-\mathbf{x}\right|}{\left|\mathbf{x}^{\prime}-\mathbf{x}\right|}=\frac{|\xi+\eta|-|\xi|}{|\xi|},
$$

where

$$
\begin{aligned}
& \xi=\mathbf{x}^{\prime}-\mathbf{x}, \\
& \boldsymbol{\eta}=\mathbf{u}^{\prime}-\mathbf{u},
\end{aligned}
$$

and $\mathbf{y}$ is the position vector in the deformed configuration, i.e. $\mathbf{y}=\mathbf{x}+\mathbf{u}$. The stretch between material points, $s$ represents the ratio of change in length between two material points after deformation to the original distance between them. $\xi$ and $\eta$ are relative position and relative displacement vectors, respectively. The $c$ parameter is a peridynamic parameter called bond constant and can be related to material constants of CCM as (Silling and Askari, 2005)

$$
c=\frac{18 \kappa}{\pi \delta^{4}}
$$

in which $\kappa$ is bulk modulus and $\delta$ is the radius of the horizon.

It is not easy to obtain an analytical solution for the PD equation of motion given in Eq. (2). Therefore, numerical techniques are generally utilised. If meshless approach is used for spatial discretisation, the solution domain is discretised into finite number of material volumes with one point representing the volume located at the center of the volume. The equation of motion of the material point $k$ can be expressed in discretised form as

$$
\rho_{(k)} \mathbf{u}_{(k)}=\sum_{j=1}^{N} \mathbf{f}\left(\mathbf{u}_{(j)}-\mathbf{u}_{(k)}, \mathbf{x}_{(j)}-\mathbf{x}_{(k)}\right) V_{(j)}+\mathbf{b}_{(k)}
$$

where $\rho_{(k)}$ is the mass density of the material point $k, N$ is the number of the family members within the horizon, $\mathbf{u}_{(k)}$ is displacement of the material point $k, \mathbf{V}_{(j)}$ is the volume of the family member point $j$, and $\mathbf{b}_{(k)}$ is the body force density acting on the material point $k$.

\section{Peridynamic Mindlin plate formulation}

The classical peridynamic theory only considers translational degrees of freedom (DOF). To derive the peridynamic equations of a Mindlin plate requires incorporation of out-of-plane deformation, $w$, and rotations, $\phi_{x}$ and $\phi_{y}$, as DOFs (see Fig. 2). 


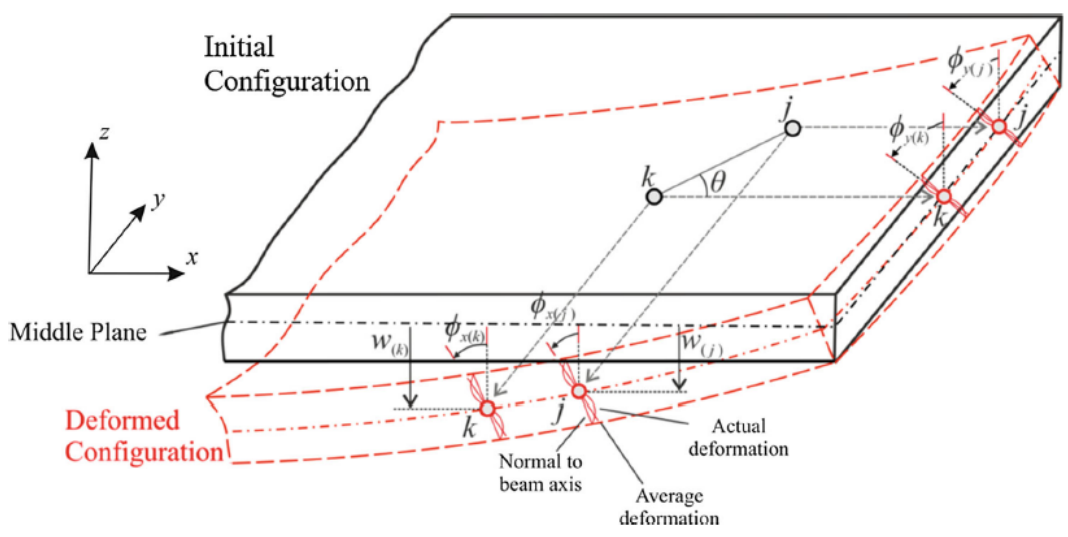

Fig. 2. Initial and deformed configurations of peridynamic Mindlin plate (Diyaroglu et. al. 2015).

Peridynamic interactions between material points can be described by introducing peridynamic transverse shear force and bending moment densities, respectively, as (Diyaroglu et al. 2015)

$$
\begin{aligned}
& \hat{f}_{(k)(j)}=c_{s} \varphi_{(k)(j)} \\
& f_{(k)(j)}=c_{b} \kappa_{(k)(j)}
\end{aligned}
$$

in which $c_{s}$ and $c_{b}$ are peridynamic parameters, i.e. bond constants, that are related with shear angle $\varphi_{(k)(j)}$ and curvature $\kappa_{(k)(j)}$, respectively, between material points $k$ and $j$. The shear angle and curvature arising from the interaction between material points $k$ and $j$ can be described as

$$
\begin{aligned}
& \varphi_{(k)(j)}=\left(\frac{w_{(j)}-w_{(k)}}{\xi_{(k)(j)}}-\frac{\phi_{(j)}+\phi_{(k)}}{2}\right) \\
& \kappa_{(k)(j)}=\frac{\phi_{(j)}-\phi_{(k)}}{\xi_{(k)(j)}}
\end{aligned}
$$

The rotations of material points $k$ and $j, \phi_{(k)}$ and $\phi_{(j)}$, can be expressed in terms of rotational DOFs, $\phi_{x}$ and $\phi_{y}$, as

$$
\begin{aligned}
& \phi_{(k)}=\phi_{x(k)} \cos \theta+\phi_{y(k)} \sin \theta \\
& \phi_{(j)}=\phi_{x(j)} \cos \theta+\phi_{y(j)} \sin \theta
\end{aligned}
$$

where $\theta$ is the orientation of the bond between material points $k$ and $j$ with respect to horizontal axis. The total kinetic energy and total potential energy of the Mindlin plate are given as

$$
T=\frac{1}{2} h \rho \sum_{k=1}^{\infty}\left(\frac{h^{2}}{12} \phi_{x(k)}^{2}+\frac{h^{2}}{12} \phi_{y(k)}^{2}+w_{(k)}^{2}\right) A_{(k)}
$$

and

$$
\begin{aligned}
U & =\sum_{k=1}^{\infty} \frac{1}{2}\left\{\sum_{j=1}^{\infty} \frac{1}{2}\left[\hat{\omega}_{(k)(j)}\left(\varphi_{(k)(j)}\right)+\hat{\omega}_{(j)(k)}\left(\varphi_{(j)(k)}\right)\right] V_{(j)}-\hat{b}_{(k)} w_{(k)}\right\} V_{(k)} \\
& +\sum_{k=1}^{\infty} \frac{1}{2}\left\{\sum_{j=1}^{\infty} \frac{1}{2}\left[\omega_{(k)(j)}\left(\kappa_{(k)(j)}\right)+\omega_{(j)(k)}\left(\kappa_{(j)(k)}\right)\right] V_{(j)}-b_{\alpha(k)} \phi_{\alpha(k)}\right\} V_{(k)}
\end{aligned}
$$


in which $h$ is the thickness of the plate, $A_{(k)}$ is the cross-sectional area of the material point $k$, and $\alpha$ can be $x$ or $y$. The three equations of motion can be obtained by substituting Eqs. (15) and (16) in Eq. (1) and replacing $\mathbf{q}_{(k)}$ with $w_{(k)}, \phi_{x(k)}$ and $\phi_{y(k)}$ as

$$
\begin{aligned}
\rho h w_{(k)} & =\sum_{j=1}^{\infty} c_{s}\left(\frac{w_{(j)}-w_{(k)}}{\xi_{(j)(k)}}-\frac{\phi_{x(j)}+\phi_{x(k)}}{2} \cos \theta-\frac{\phi_{y(j)}+\phi_{y(k)}}{2} \sin \theta\right) V_{(j)}+\hat{b}_{(k)} h, \\
\frac{\rho h^{3}}{12} \phi_{x(k)}= & c_{b} \sum_{j=1}^{\infty}\left(\frac{\phi_{x(j)}-\phi_{x(k)}}{\xi_{(j)(k)}} \cos \theta+\frac{\phi_{y(j)}-\phi_{y(k)}}{\xi_{(j)(k)}} \sin \theta\right) \cos \theta V_{(j)}+ \\
& \frac{1}{2} c_{s} \sum_{j=1}^{\infty} \xi_{(j)(k)}\left(\frac{w_{(j)}-w_{(k)}}{\xi_{(j)(k)}}-\frac{\phi_{x(j)}+\phi_{x(k)}}{2} \cos \theta-\frac{\phi_{y(j)}+\phi_{y(k)}}{2} \sin \theta\right) \cos \theta V_{(j)}+b_{x(k)} h
\end{aligned}
$$

and

$$
\begin{aligned}
\frac{\rho h^{3}}{12} \phi_{y(k)}= & c_{b} \sum_{j=1}^{\infty}\left(\frac{\phi_{x(j)}-\phi_{x(k)}}{\xi_{(j)(k)}} \cos \theta+\frac{\phi_{y(j)}-\phi_{y(k)}}{\xi_{(j)(k)}} \sin \theta\right) \sin \theta V_{(j)}+ \\
& \frac{1}{2} c_{s} \sum_{j=1}^{\infty} \xi_{(j)(k)}\left(\frac{w_{(j)}-w_{(k)}}{\xi_{(j)(k)}}-\frac{\phi_{x(j)}+\phi_{x(k)}}{2} \cos \theta-\frac{\phi_{y(j)}+\phi_{y(k)}}{2} \sin \theta\right) \sin \theta V_{(j)}+b_{y(k)} h
\end{aligned}
$$

Peridynamic parameters $c_{s}$ and $c_{b}$ can be expressed in terms of elastic modulus, $E$ as

$$
c_{s}=\frac{9 E}{4 \pi \delta^{3}} k_{s}^{2}
$$

and

$$
c_{b}=\frac{3 h^{2} E}{4 \pi \delta^{3}}
$$

where $k_{s}$ represent the shear correction factor and the value of $\pi^{2} / 12$ is used for most plates (Stephen, 1997). Please note that peridynamic Mindlin equations given in Eqs. (17-19) are in the form of infinite summations with infinitely small volumes. This means that these infinite summations can also be written in integral form as in Eq. (2). Although in peridynamics it is possible to define that all material points can interact with each other, in order make computations feasible, an influence region, horizon, is introduced so that only material points inside the horizon can interact with each other. If a uniform discretization is utilized, the size of the horizon is usually chosen as three times of the discretization size. Please also note that the main difference between the classical Mindlin equations and peridynamic Mindlin equations is their mathematical form. Classical Mindlin equations are in the form of partial differential equations whereas the peridynamic equations are in the form of integrations or summations and do not contain spatial derivatives.

\section{Implementation of peridynamic Mindlin plate formulation in ANSYS}

In this study, the numerical implementation was done by using the commercial finite element software, ANSYS version R19.2 and following the approach presented in Yang et al. (2018). Although 
classical finite element method and peridynamics has fundamental differences, it is possible to implement peridynamic formulation in a commercial finite element software by representing peridynamic interactions with traditional finite elements (see Fig. 3) and calibrating parameters of finite element analysis with parameters of peridynamic analysis. Peridynamic Mindlin formulation presented in the previous section can be represented by using BEAM4 elements since this element has both translational and rotational DOFs and is based on Timoshenko beam element.

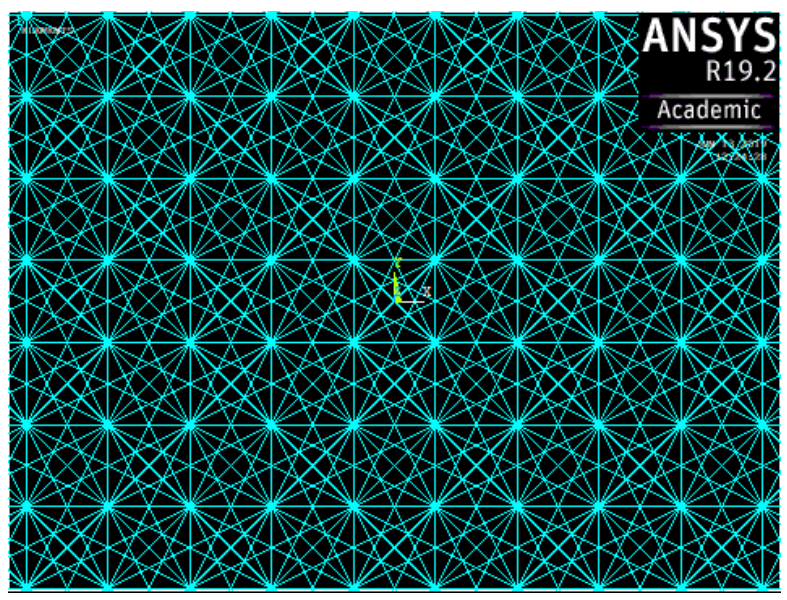

Fig. 3. Material points (represented by nodes) and peridynamic interactions between material points (represented by beam elements) implemented in ANSYS.

To represent peridynamic interactions in finite element framework, peridynamic equations of motion given in Eqs. (17-19) can be rewritten for finite number of interactions by multiplying all equations with the volume of the material point $k$, dividing with the plate thickness, $h$, and using the shear angle and curvature definitions given in Eqs. (11) and (12) as

$$
\begin{gathered}
\rho V_{(k)} w_{(k)}=\sum_{j=1}^{N} \frac{c_{s}}{h} \varphi_{(k)(j)} V_{(j)} V_{(k)}+\hat{b}_{(k)} h, \\
\rho V_{(k)} \frac{I}{A} \phi_{x(k)}=\sum_{j=1}^{N} \frac{c_{b}}{h} \kappa_{(k)(j)} V_{(j)} V_{(k)}+\frac{1}{2} \sum_{j=1}^{N} \frac{c_{s}}{h} \varphi_{(k)(j)} \xi_{(j)(k)} \cos \theta V_{(j)} V_{(k)}+b_{x(k)} V_{(k)},
\end{gathered}
$$

and

$$
\rho V_{(k)} \frac{I}{A} \phi_{y(k)}=\sum_{j=1}^{N} \frac{c_{b}}{h} \kappa_{(k)(j)} V_{(j)} V_{(k)}+\frac{1}{2} \sum_{j=1}^{N} \frac{c_{s}}{h} \varphi_{(k)(j)} \xi_{(j)(k)} \sin \theta V_{(j)} V_{(k)}+b_{y(k)} V_{(k)}
$$

where $N$ is the number of the material points within the horizon. Based on Eqs. (22-24), peridynamic bond forces and moments between material points $k$ and $j$ can be expressed as

$$
\begin{aligned}
& \hat{f}_{(k)(j)}=\frac{c_{s}}{h} V_{(j)} V_{(k)} \varphi_{(k)(j)} \\
& f_{(k)(j)}=\frac{c_{b}}{h} V_{(j)} V_{(k)} \kappa_{(k)(j)}
\end{aligned}
$$

According to Classical Continuum Mechanics, the force and bending moment of Timoshenko beam can 
be described as

$$
\begin{aligned}
& \hat{f}_{(k)(j)}=k_{s} A G_{(k)(j)} \varphi_{(k)(j)} \\
& f_{(k)(j)}=E_{(k)(j)} I_{y} \kappa_{(k)(j)}
\end{aligned}
$$

where $G_{(k)(j)}$ and $E_{(k)(j)}$ represent peridynamic shear modulus and Young's modulus of the bond between material points $k$ and $j . k_{s}, A$ and $I_{y}$ represent shear correction factor, cross-sectional area and moment of inertia of the beam, respectively. Note that these three parameters, i.e. $k_{s}, A$ and $I_{y}$, are geometrical properties that are calculated based on the distance between material points and plate thickness. Therefore, these values cannot be used as calibration parameters. On the other hand, $G_{(k)(j)}$ and $E_{(k)(j)}$ can serve as calibration parameters. By equating peridynamic and CCM forces and moments given in Eqs. $(25,26)$ and $(27,28)$, respectively, calibrated shear modulus and Young's modulus values to be used in finite element analysis can be obtained as

$$
G_{(k)(j)}=\frac{c_{s}}{k_{s} A h} V_{(j)} V_{(k)}
$$

and

$$
E_{(k)(j)}=\frac{c_{b}}{I_{y} h} V_{(j)} V_{(k)}
$$

Please note that ANSYS is only used to solve peridynamic Mindlin equations given in Eqs. (22-24). Although finite elements are utilized, they are mainly used to represent the peridynamic forces and moments between material points. The main motivation of following such an approach is to utilize the advanced computational features of commercial finite element software packages since they use efficient solvers and allows parallel computing without putting extra effort. Moreover, complex analysis can be easily performed such as modal analysis (as in the current study) and geometrical nonlinear analysis because their solution process are already available within the commercial software package. Please also note that such an approach also introduces additional feature to commercial finite element packages, so that a finite element user can perform peridynamic analysis without the requirement of writing a new inhouse peridynamic code.

\section{Free vibration analysis of cracked plates}

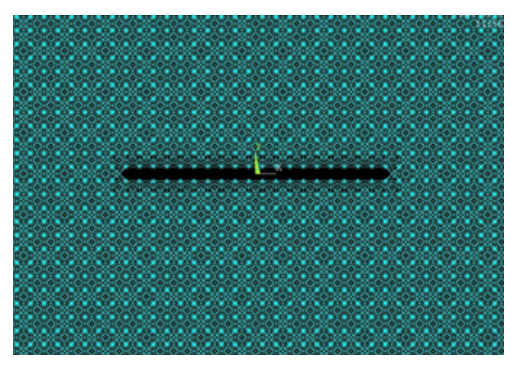

Fig. 4. Creation of cracks in ANSYS without including elements (interactions) passing through the crack surface.

In this section, the free vibration analysis of cracked plates is performed by using the peridynamic 
model implemented in ANSYS. BEAM4 element in ANSYS represents each bond between material points and the MASS21 element represents the mass of each material point. As shown in Fig. 4, cracks are created without including elements (interactions) passing through the crack surface. Please note that it is pretty straightforward to define cracks and any number of cracks can exist in the solution domain and they can easily interact with each other. Other existing methods can encounter difficulties especially analyzing multiple crack cases. Various crack configurations are considered to investigate the effect of crack length and crack orientation. Peridynamic results are compared with experimental, theoretical and numerical results provided by Krawczuk (1993), Barton (1951) and Qian et al. (1991).

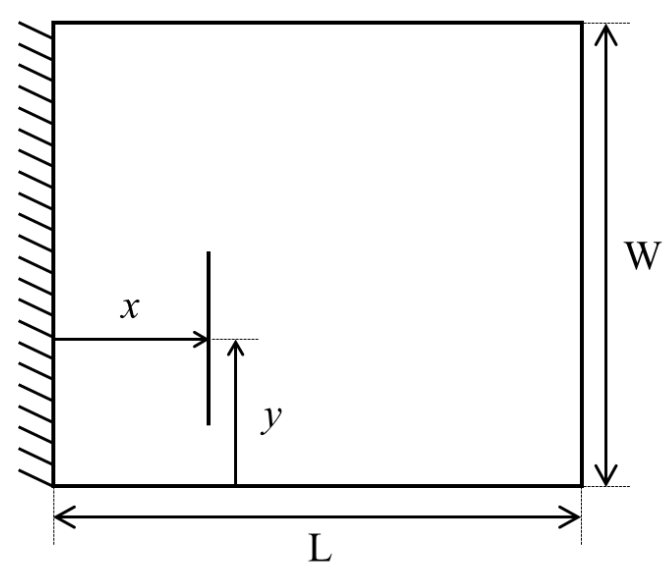

Fig. 5. The geometry of a cantilever plate with a crack.

\subsection{Verification cases}

For the numerical analysis, a cantilever plate with in-plane dimensions of $240 \times 240 \mathrm{~mm}^{2}$ and thickness of $2.75 \mathrm{~mm}$ is considered as shown in Fig. 5. The plate is made of aluminium with Young's modulus of $67 \mathrm{GPa}$, Poisson's ratio of $1 / 3$ and mass density of $2800 \mathrm{~kg} / \mathrm{m}^{3}$. First, natural frequencies of an intact plate without crack are obtained for verification purposes. As shown in Table 1, peridynamic results are compared with numerical and theoretical results of Krawczuk (1993) and Barton (1951), respectively, and the finite element results obtained using ANSYS shell element. The natural frequencies from all four different approaches are similar to each other and the differences between FE and peridynamic results are smaller than $3 \%$.

Next, the values for relative change of natural frequencies of cracked plates with respect to intact plates are obtained and compared with values from Krawczuk (1993) and Qian et al. (1991). The crack is parallel to the fixed edge with a length of $34 \mathrm{~mm}$ and its centre is located $90 \mathrm{~mm}$ apart from the lower right corner of the plate, i.e. $x=y=90 \mathrm{~mm}$. Based on the results given in Table 2, a good agreement between peridynamic results and the numerical, theoretical and experimental results from the literature is obtained. Note that the natural frequencies of the cracked plate given in this table are normalized with respect to the natural frequencies of the intact plate. Therefore, it can be concluded that the current peridynamic model provides reliable natural frequencies and can be used for the analysis of other configurations. 
Table 1. Validation of peridynamic result with other methods of intact plate.

\begin{tabular}{llllll}
\hline \hline Mode & $\begin{array}{l}\text { Krawczuk } \\
(1993)\end{array}$ & $\begin{array}{l}\text { Barton } \\
(1951)\end{array}$ & $\begin{array}{l}\text { FEM } \\
{[\mathrm{Hz}]}\end{array}$ & $\begin{array}{l}\text { Peridynamics } \\
{[\mathrm{Hz}]}\end{array}$ & $\begin{array}{l}\% \\
\text { Diff }\end{array}$ \\
\hline 1 & 41.49 & 40.39 & 39.36 & 40.452 & 2.70 \\
2 & 99.87 & 96.94 & 94.89 & 101.10 & 1.92 \\
3 & 255.91 & 242.36 & 239.92 & 249.77 & 1.31 \\
\hline \hline
\end{tabular}

Table 2. Comparison of relative changes in natural frequencies of cracked plate.

\begin{tabular}{lllll}
\hline Mode & $\begin{array}{l}\text { Krawczuk } \\
(1993)\end{array}$ & $\begin{array}{l}\text { Qian et al. } \\
(1991)\end{array}$ & $\begin{array}{l}\text { Qian et al. } \\
(1991) \\
\text { numerical }\end{array}$ & theoretical \\
\hline 1 & 0.9891 & 0.9931 & 0.9917 & 0.9909 \\
2 & 0.9985 & 0.9989 & 0.9981 & 0.9978 \\
3 & 0.9826 & 0.9837 & 0.9807 & 0.9899 \\
\hline \hline
\end{tabular}

\subsection{Demonstration cases}

After verifying the current formulation, an analysis to investigate the effect of crack length, crack angle (orientation) and plate thickness is performed for a centrally cracked plate. The material properties are the same as in the previous case. The plate is subjected to a clamped-clamped condition (CFCF) and the results are summarised in Table 3. As can be seen in these results, natural frequencies increase as the thickness of the plate increases. On the other hand, the crack length increase yields reduction in natural frequency values. Moreover, although natural frequency is insensitive to crack angle change for smaller cracks, i.e. $c / W=0.1$, natural frequency values increase as the crack angle increases. Comparing plate thickness, crack length and crack angle, plate thickness has more influence on natural frequency values. Fig. 6 shows the first five-mode shapes for three cases of crack angle $\left(0^{\circ}, 30^{\circ}\right.$ and $\left.60^{\circ}\right)$ for the centrally cracked plate, respectively. As shown in this figure, mode shapes change as the crack angle increases. In particular, there are significant differences in modes of fourth and higher. 
Table 3. Peridynamic results for natural frequencies of the cracked plate (CFCF).

\begin{tabular}{|c|c|c|c|}
\hline $\begin{array}{l}\text { Thickness } \\
{[\mathrm{mm}]}\end{array}$ & $\begin{array}{l}\text { Crack } \\
\text { length } \\
\text { ratio } \\
(c / W)\end{array}$ & $\begin{array}{l}\text { Crack } \\
\text { angle } \\
(\alpha)\end{array}$ & $\begin{array}{l}\text { Peridynamics } \\
{[\mathrm{Hz}]}\end{array}$ \\
\hline \multirow[t]{9}{*}{8} & 0.1 & $0^{\circ}$ & 706.18 \\
\hline & & $30^{\circ}$ & 707.99 \\
\hline & & $60^{\circ}$ & 709.99 \\
\hline & 0.3 & $0^{\circ}$ & 676.32 \\
\hline & & $30^{\circ}$ & 686.03 \\
\hline & & $60^{\circ}$ & 703.15 \\
\hline & 0.5 & $0^{\circ}$ & 631.05 \\
\hline & & $30^{\circ}$ & 657.55 \\
\hline & & $60^{\circ}$ & 695.31 \\
\hline \multirow[t]{9}{*}{10} & 0.1 & $0^{\circ}$ & 874.09 \\
\hline & & $30^{\circ}$ & 874.30 \\
\hline & & $60^{\circ}$ & 879.50 \\
\hline & 0.3 & $0^{\circ}$ & 837.09 \\
\hline & & $30^{\circ}$ & 848.17 \\
\hline & & $60^{\circ}$ & 870.63 \\
\hline & 0.5 & $0^{\circ}$ & 780.83 \\
\hline & & $30^{\circ}$ & 812.47 \\
\hline & & $60^{\circ}$ & 860.72 \\
\hline \multirow[t]{9}{*}{12} & 0.1 & $0^{\circ}$ & 1040.0 \\
\hline & & $30^{\circ}$ & 1038.9 \\
\hline & & $60^{\circ}$ & 1045.87 \\
\hline & 0.3 & $0^{\circ}$ & 994.86 \\
\hline & & $30^{\circ}$ & 1007.3 \\
\hline & & $60^{\circ}$ & 1035.1 \\
\hline & 0.5 & $0^{\circ}$ & 927.95 \\
\hline & & $30^{\circ}$ & 964.54 \\
\hline & & $60^{\circ}$ & 1022.98 \\
\hline
\end{tabular}


$0^{\circ}$
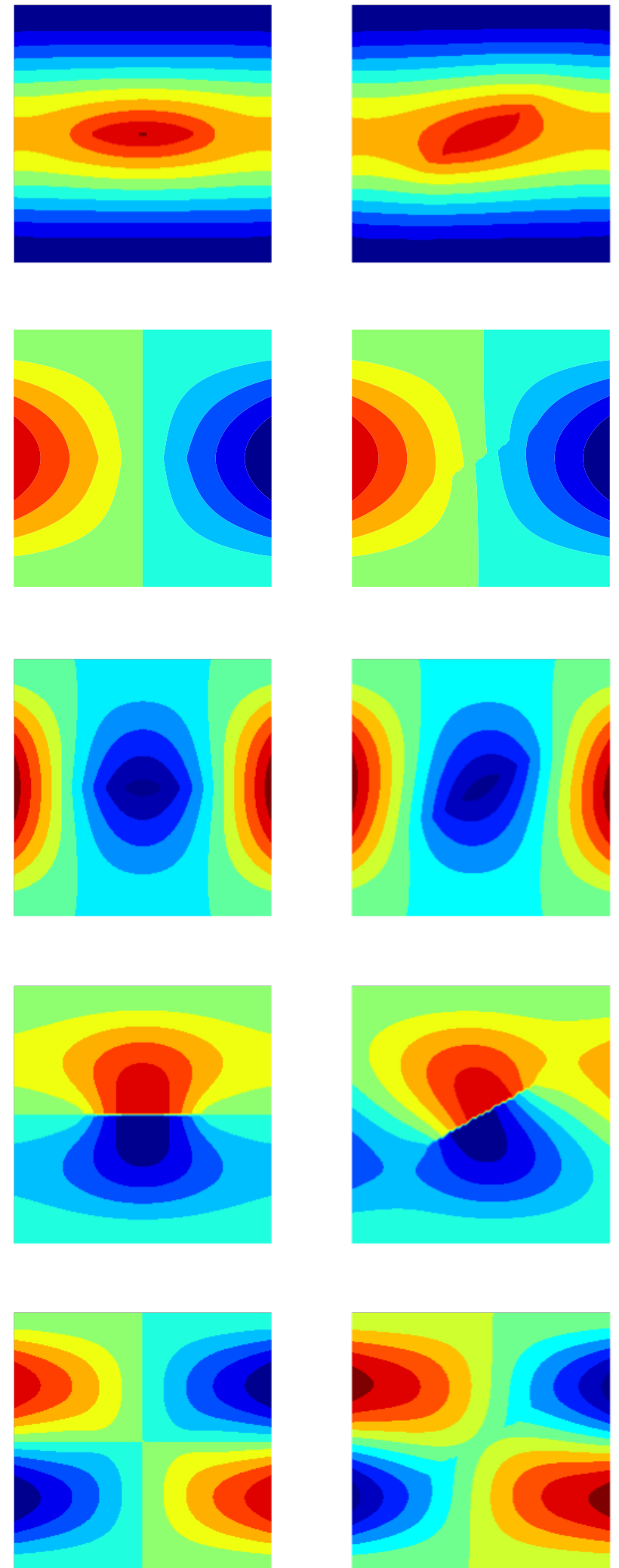
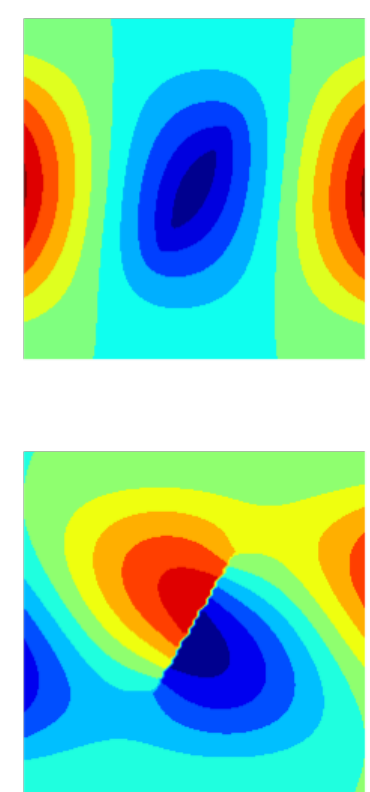

$60^{\circ}$
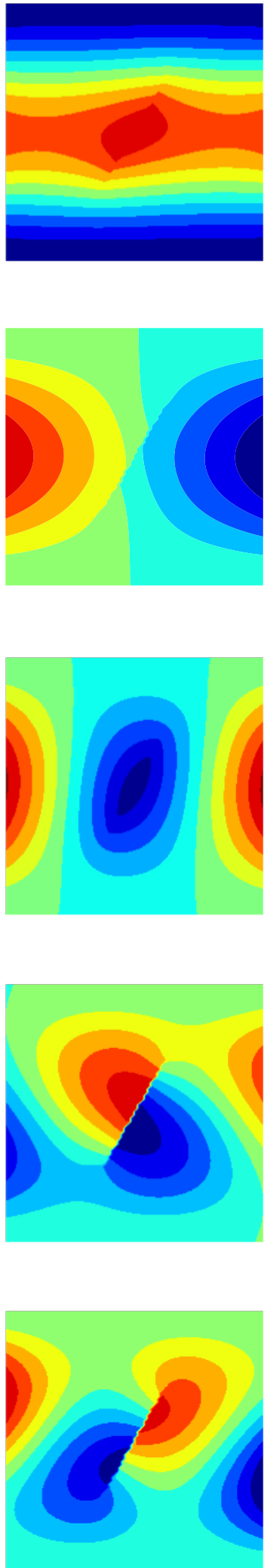

Fig. 6. First five vibration mode shapes of central cracked plate (CFCF)

(Mode: from top to bottom; 1st, 2nd, 3rd, 4th and 5th) (Crack angle: from left to right; $0^{\circ}, 30^{\circ}$ and $60^{\circ}$ ) 


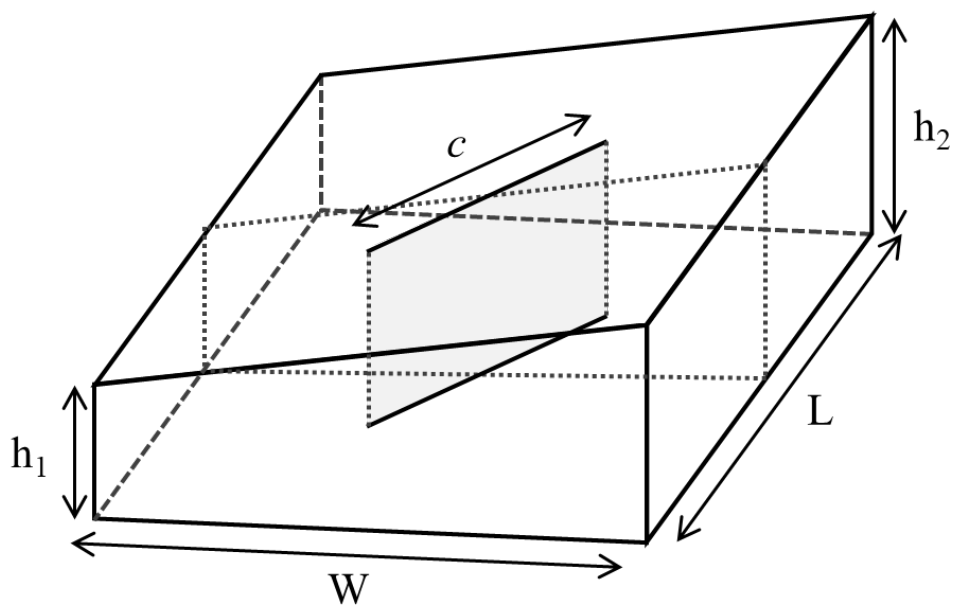

Fig. 7. The geometry of a plate with a variable thickness along the length of the plate.

Next, the free vibration analysis was performed for a centrally cracked plate with linearly increased thickness. The in-plane dimensions of the plate are same as in the previous case. As shown in Fig. 7, the plate has a different thickness in the direction of the width. The $h_{1}$ and $h_{2}$ parameters are the smallest and largest thicknesses of the plate, respectively. The thickness ratio $\left(h_{2} / h_{1}\right)$ is specified as 2 with the right side of the plate becoming the thick side. Fig. 8 shows the mode shapes for the plate with linearly varying thickness. Overall, it can be seen that the centre of each vibration mode shape is tilted to the left which is the thin side of the plate. Other than this difference, it has similar mode shapes as the constant thickness case demonstrated in Fig. 6.

\section{Conclusions}

In this study, a free vibration analysis of cracked plates was performed by using peridynamics. A commercial finite element software, ANSYS was utilized for numerical implementation of peridynamics. Peridynamic formulation was verified by considering a cantilever plate with and without a crack. Peridynamic results were compared against numerical, theoretical and experimental results available in the literature and a good agreement was obtained between different approaches. After verifying the formulation, it was utilised to investigate the effect of crack length, crack orientation and plate thickness on the natural frequency values for a centrally crack plate with CFCF boundary conditions. After considering many different configuration, it was observed that natural frequencies increase as the plate thickness increases. This is expected since increase in thickness will increase stiffness which will then increase the natural frequency. On the hand, increase in crack length decreases the natural frequency values. This is also expected since defects like cracks decrease the stiffness which will then decrease the natural frequency. Regarding the effect of crack orientation, although smaller cracks have minimal effect on natural frequency values, natural frequency values increase as the crack orientation angle increases. Amongst the three factors, the most dominant factor was found to be the plate thickness. Mode shapes were also investigated for different crack orientations. It was shown that the effect of crack orientation increases as the mode number increases. Finally, a centrally cracked plate with linearly increased thickness was considered. It was found that the center of each vibration mode shape is tilted to the thin side of the plate. Based on the validation and demonstration cases considered in this study, it can be concluded that peridynamics can be a suitable alternative for the free vibration analysis of cracked plates. 
As a future study, free vibration analysis of cracked stiffened plates, which are widely used in ships and offshore structures, can be investigated by using the developed methodology. Moreover, free vibration analysis of a plate with a large number of cracks can also be studied which is a very good example to demonstrate the advantage of peridynamics with respect to some other existing approaches.

$0^{\circ}$
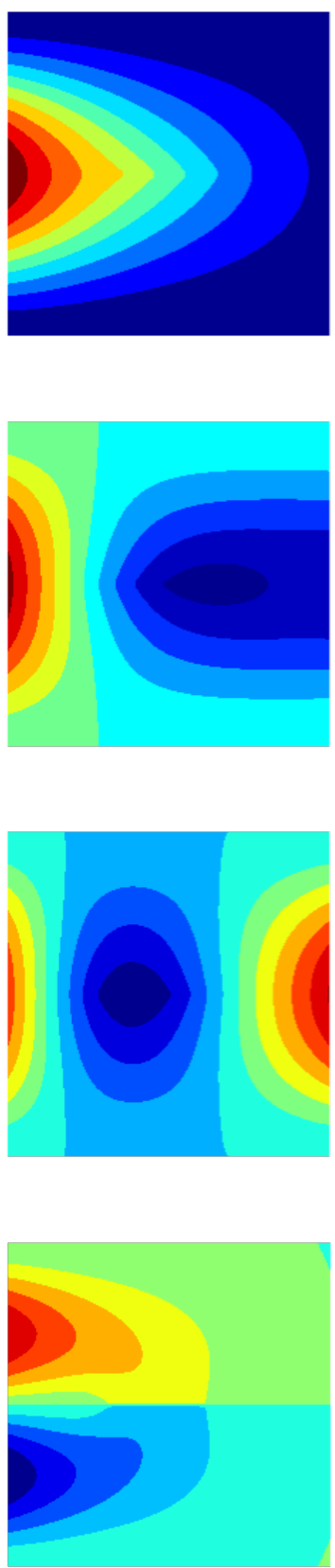

$30^{\circ}$
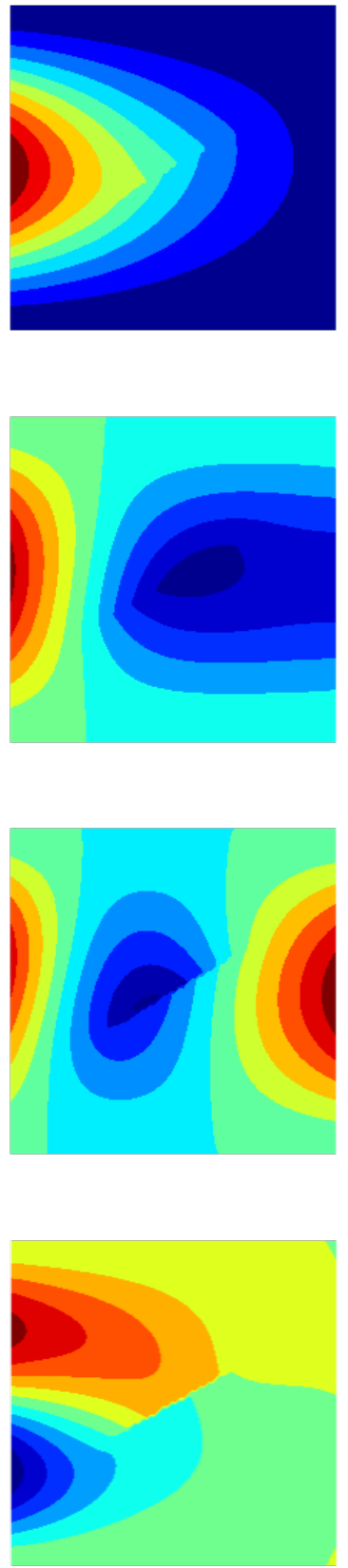
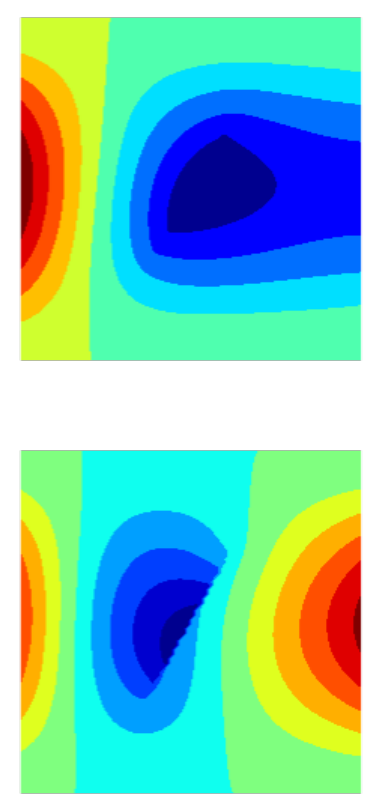

$60^{\circ}$
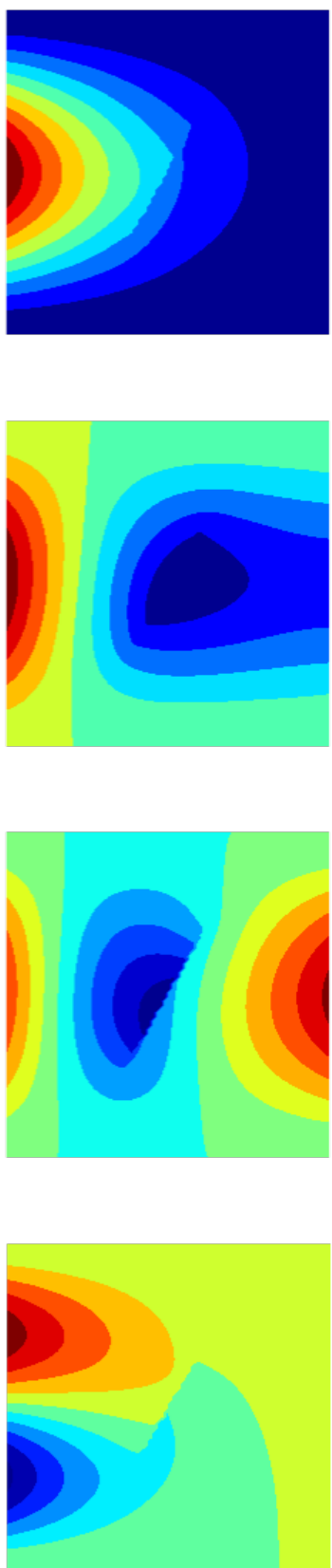

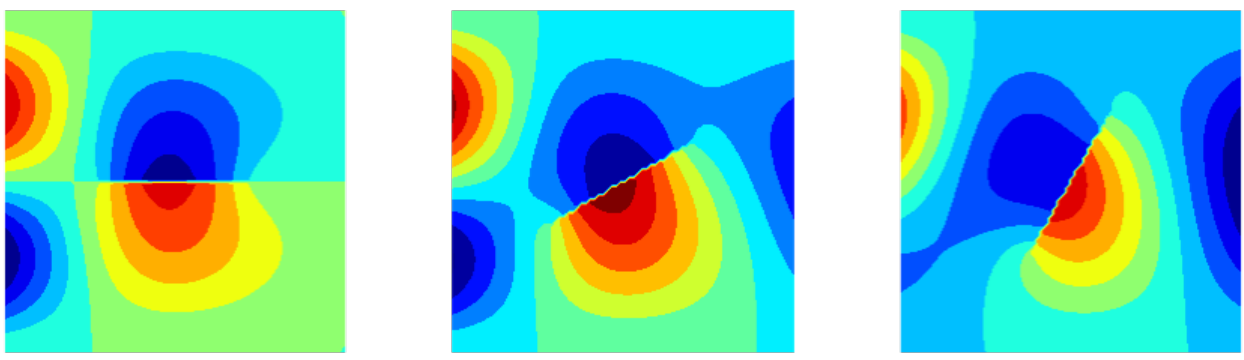

Fig. 8. First five vibration mode shapes of a variable thickness cracked plate (CFCF)

(Mode: from top to bottom; 1st, 2nd, 3rd, 4th and 5th)(Crack angle: from left to right; $0^{\circ}, 30^{\circ}$ and $60^{\circ}$ )

\section{Acknowledgements}

This research was funded and conducted under "the Competency Development Program for Industry Specialists" of the Korean Ministry of Trade, Industry and Energy (MOTIE), operated by Korean Institute for Advancement of Technology (KIAT). (No. N0001287, HRD program for Korea-UK Global Engineer Education Program for Offshore Plant).

\section{References}

Azam MS, Ranjan V, Kumar B. 2017. Free Vibration Analysis of Rhombic Plate with Central Crack. International Journal of Acoustics and Vibration, 22(4): 481-492.

Barton M. 1951. Vibration of rectangular and skew cantilever plates. Journal of Applied Mechanics Transactions of the ASME. 18: 129-134.

Diyaroglu C, Oterkus E, Oterkus S and Madenci E. 2015. Peridynamics for bending of beams and plates with transverse shear deformation. International Journal of Solids and Structures. (69-70): 152-168.

Doan DH, Van Do T, Pham PM, Duc ND. 2019. Validation simulation for free vibration and buckling of cracked Mindlin plates using phase-field method. Mechanics of Advanced Materials and Structures. 26(12): 1018-1027.

Gupta AK, Vimal J, Chaturvedi V. 2018. Free vibration analysis of a centre-cracked plate at various orientations through finite element method. International Journal of Composite and Constituent Materials, 4(1): 5-18.

Guski V, Verestek W, Oterkus E, Schmauder S. 2020. Microstructural investigation of plasma sprayed ceramic coatings using peridynamics. Journal of Mechanics, 36(2): 183-196.

Hosseini-Hashemi S, Gh HR, DT HR. 2010. Exact free vibration study of rectangular Mindlin plates with all-over part-through open cracks. Computers \& Structures, 88(17-18): 1015-1032.

Huang CS, Leissa AW, Li RS. 2011. Accurate vibration analysis of thick, cracked rectangular plates. Journal of Sound and Vibration, 330(9): 2079-2093.

Imachi M, Tanaka S, Bui TQ, Oterkus S, Oterkus E. 2019. A computational approach based on ordinary state-based peridynamics with new transition bond for dynamic fracture analysis. Engineering Fracture Mechanics, 206: 359-374.

Imachi M, Tanaka S, Ozdemir M, Bui TQ, Oterkus S, Oterkus, E., 2020. Dynamic crack arrest analysis by ordinary state-based peridynamics. International Journal of Fracture, 221(2): 155-169.

Javili A, McBride AT, Steinmann P. 2019. Continuum-kinematics-inspired peridynamics. Mechanical problems. Journal of the Mechanics and Physics of Solids, 131: 125-146. 
Javili A, Morasata R, Oterkus E, Oterkus S. 2019. Peridynamics review. Mathematics and Mechanics of Solids, 24(11): 3714-3739.

Joshi PV, Jain NK, Ramtekkar GD. 2015. Effect of thermal environment on free vibration of cracked rectangular plate: An analytical approach. Thin-Walled Structures, 91: 38-49.

Joshi PV, Gupta A, Jain NK, Salhotra R, Rawani AM, Ramtekkar GD. 2017. Effect of thermal environment on free vibration and buckling of partially cracked isotropic and FGM micro plates based on a non classical Kirchhoff's plate theory: An analytical approach. International Journal of Mechanical Sciences, 131: 155-170.

Krawczuk M. 1993. Natural vibrations of rectangular plates with a through crack. Archieve of Applied Mechanics. 63(7): 491-504.

Li M, Lu W, Oterkus E, Oterkus S. 2020. Thermally-induced fracture analysis of polycrystalline materials by using peridynamics. Engineering Analysis with Boundary Elements, 117: 167-187.

Madenci E, Oterkus E. 2014. Peridynamic Theory and Its Applications. New York, NY: Springer. Nguyen, C.T. and Oterkus, S., 2019. Peridynamics formulation for beam structures to predict damage in offshore structures. Ocean Engineering, 173, pp.244-267.

Nguyen-Thanh N, Li W, Zhou K. 2018. Static and free-vibration analyses of cracks in thin-shell structures based on an isogeometric-meshfree coupling approach. Computational Mechanics, 62(6):1287-1309.

Nguyen-Thoi T, Rabczuk T, Lam-Phat T, Ho-Huu V, Phung-Van P. 2014. Free vibration analysis of cracked Mindlin plate using an extended cell-based smoothed discrete shear gap method (XCS-DSG3). Theoretical and Applied Fracture Mechanics, 72: 150-163.

Ni T, Zaccariotto M, Zhu QZ, Galvanetto U. 2019. Static solution of crack propagation problems in Peridynamics. Computer Methods in Applied Mechanics and Engineering, 346: 126-151.

Ozdemir M, Kefal A, Imachi M, Tanaka S, Oterkus E. 2020. Dynamic fracture analysis of functionally graded materials using ordinary state-based peridynamics. Composite Structures, p.112296.

Qian GL, Gu SN, Jiang JS. 1991. A finite element model of cracked plates and application to vibration problems. Computers \& Structures. 39(5): 483-487.

Shahverdi H, Navardi MM. 2017. Free vibration analysis of cracked thin plates using generalized differential quadrature element method. Structural Engineering and Mechanics, 62(3): 345-355.

Silling SA, Askari E. 2005. A meshfree method based on the peridynamic model of solid mechanics. Computers \& Structures. 83(17-18): 1526-1535.

Soni S, Jain NK, Joshi PV. 2018. Vibration analysis of partially cracked plate submerged in fluid. Journal of Sound and Vibration, 412: 28-57.

Stephen N. 1997. Mindlin plate theory: best shear coefficient and higher spectra validity. Journal of Sound and Vibration. 202(4): 539-553.

Vazic B, Oterkus E, Oterkus, S. 2020. In-Plane and Out-of Plane Failure of an Ice Sheet using Peridynamics. Journal of Mechanics, 36(2): 265-271.

Wang B, Oterkus S, Oterkus, E. 2020. Determination of horizon size in state-based peridynamics.

Continuum Mechanics and Thermodynamics: 1-24.

Wang X, Kulkarni SS, Tabarraei A. 2019. Concurrent coupling of peridynamics and classical elasticity for elastodynamic problems. Computer methods in applied mechanics and engineering, 344: 251-275. $\mathrm{Xu}$ Z, Chen W. 2017. Vibration analysis of plate with irregular cracks by differential quadrature finite element method. Shock and Vibration, 2017: 2073453. 
Xue J, Wang Y. 2019. Free vibration analysis of a flat stiffened plate with side crack through the Ritz method. Archive of Applied Mechanics, 89(10): 2089-2102.

Yang G, Hu D, Han X, Ma G. 2017. An extended edge-based smoothed discrete shear gap method for free vibration analysis of cracked Reissner-Mindlin plate. Applied Mathematical Modelling, 51: 477504.

Yang Z, Oterkus E, Nguyen CT and Oterkus S. 2019. Implementation of peridynamic beam and plate formulations in finite element framework. Continuum Mechanics and Thermodynamics. 31(1): 301-315. Ye LY, Guo CY, Wang C, Wang CH, Chang X. 2019. Peridynamic solution for submarine surfacing through ice. Ships and Offshore Structures: 1-15.

Yu T, Bui TQ, Liu P, Hirose S. 2016. A stabilized discrete shear gap extended finite element for the analysis of cracked Reissner-Mindlin plate vibration problems involving distorted meshes.

International Journal of Mechanics and Materials in Design, 12(1): 85-107.

Zeng HC, Huang CS, Leissa AW, Chang MJ. 2016. Vibrations and stability of a loaded side-cracked rectangular plate via the MLS-Ritz method. Thin-Walled Structures, 106: 459-470.

Zhang Y. 2018. Free vibration analysis of cracked rectangular plates using the discrete singular convolution method. MSc Thesis. Hong Kong Polytechnic University. 